\title{
OS PRIMEIROS MESES NA DANÇA RELACIONAL MÃE-BEbÉ(S): DESAFIOS DECORRENTES DA SITUAÇÃOO GEMELAR
}

\author{
Sofia Veiga \\ Escola Superior de Educação \\ Instituto Politécnico do Porto \\ sofiaveiga@ese.ipp.pt \\ Ana Bertão \\ Escola Superior de Educação \\ Instituto Politécnico do Porto \\ anabertao@ese.ipp.pt \\ Madalena Alarcão \\ Faculdade de Psicologia e de Ciências da Educação \\ Universidade de Coimbra \\ madalena.alarcao@uc.pt
}

Fecha de Recepción: 2 Septiembre 2019

Fecha de Admisión: 25 Septiembre 2019

\section{RESUMEN}

É decorrente das competências percetivas e relacionais da mãe e do bebé, e da sua dança relacional, que 0 comportamento de vinculação se vai gradualmente desenvolvendo; e é da qualidade desta dança relacional que dependerá, a posteriori, o solo individual e a articulação de dois desenvolvimentos interligados, mas independentes. Ora, no caso das mães gemelares, estas têm de estabelecer relações individuais com duas ou mais crianças, com a mesma idade, tendo em conta as suas necessidades idênticas, assim como as suas distintas personalidades, necessidades e padrões de interação.

A pesquisa que se apresenta procurou investigar a existência de uma relação, nos seis primeiros meses de vida, entre 0 tipo de ligação vinculativa mãe-bebés gémeos e a diferenciação que a mãe faz dos mesmos.

Em termos de procedimentos, foram realizadas três entrevistas semi diretivas a quarenta mães gemelares, de tipo clínico, uma após o parto e as outras aos três e aos seis meses. Para a análise dos dados, optou-se por uma metodologia quantitativa, utilizando uma análise correlacional das variáveis.

A análise realizada concluiu sobre a não existência de uma relação clara entre as duas variáveis. Não obstante, a qualidade da relação mãe-bebés parece ser afetada por variáveis como: a vivência materna do parto relativamente a si e aos bebés; as complicações clínicas durante o parto; a primeira imagem materna dos bebés reais e a estadia dos bebés na incubadora; a capacidade de a mãe integrar o bebé real no bebé imaginário; a perceção materna das (des)vantagens da situação gemelar e da sua competência para lidar adequadamente com ambas as crianças e com cada uma, em particular. 


\section{OS PRIMEIROS MESES NA DANÇA RELACIONAL MÃE-BEBÉ(S): DESAFIOS DECORRENTES DA SITUAÇÃO GEMELAR}

Compreende-se, assim, a importância de as mães gemelares serem apoiadas, particularmente no período pós-parto, já que têm de enfrentar uma multiplicidade de desafios que podem afetar os processos de vinculação e de separação-individuação.

Palabras clave: vinculação; separação-individuação; díade mãe-gémeos

\section{ABSTRACT}

First months in baby mother relationship: challenges from gemelar situation. It is due to the mother and baby's perceptive and relational skills, and their relational dance, that the bonding behavior gradually develops; and it is the quality of this relational dance that will depend, a posteriori, on the individual solo and the articulation of two interconnected but independent developments. However, in the case of twin mothers, they have to establish individual relationships with two or more children of the same age, taking into account their identical needs as well as their different personalities, needs and patterns of interaction.

The research presented here seeks to investigate the existence of a relationship, within the first six months of life, between the type of mother-twin bond binding and the mother's differentiation.

In terms of procedures, three semi-directive interviews were conducted with forty clinical twin mothers, one after delivery and the other both three and six months. For data analysis, a quantitative methodology was chosen, using a correlational analysis of the variables.

The analysis concluded that there was no clear relationship between the two variables. Nevertheless, the quality of the mother-infant relationship seems to be affected by variables such as the maternal experience of childbirth in relation to yourself and babies; clinical complications during childbirth; the first maternal image of the real babies and the babies' stay in the incubator; the ability of the mother to integrate the real baby into the imaginary baby; the maternal perception of the (dis) advantages of the twin situation and their competence to deal adequately with both children and each in particular.

Thus, it is understood the importance of twin mothers being supported, particularly in the postpartum period, as they face a multitude of challenges that can affect the processes of attachment and separation-individuation.

Keywords: attachment; separation-individuation; mother-twin dyad

\section{INTRODUCCIÓN}

Após os desafios vivenciados durante a gravidez, particularmente no concerne à aceitação da situação gemelar e à preparação para uma realidade (física e psicológica) que integre duas crianças, novos reptos são colocados à mulher com 0 nascimento dos filhos.

A uma gravidez de risco sucede-se, frequentemente, um parto de alto risco (Blanc et al., 1988) e a vinda ao mundo de crianças com um risco de morbilidade e mortalidade mais elevado, uma vez que os gémeos nascem frequentemente prematuros, com baixo peso e com problemas médicos, pese embora a melhoria da assistência e cuidados médicos das últimas décadas (Patrício, 1993).

Após o parto, a mãe deverá enfrentar com segurança os aspetos concretos da situação gemelar: descobrir imediatamente os gestos indispensáveis aos cuidados e à saúde de dois bebés e de cada um, em particular. Ora, esta tarefa é muitas vezes dificultada quer pelos problemas médicos e alimentares dos gémeos, quer pela fadiga excessiva das mulheres. Face ao carácter absorvente destes cuidados, certas mães recorrem à ajuda sistemática do pessoal técnico e auxiliar, preferindo repousar durante a estadia no hospital para melhor enfrentar o regresso a casa. Esta situação é particularmente sentida, como vimos, quando nascem crianças prematuras e têm necessidade de permanecer em incubadora. Após a saída da maternidade, as mães gemelares recorrem sobretudo à ajuda familiar, sendo o pai das crianças a sua principal fonte de suporte (Anderson \& Anderson, 1990).

A gemelaridade pode confrontar o sistema familiar com múltiplas vulnerabilidades e dificuldades ao nível da gestão quotidiana. A sobrecarga de trabalho na realização de rotinas diversas e inacabáveis, bem como a privação de horas de sono, têm sido alguns dos fatores identificados em famílias de gémeos geradores de stresse e ansiedade (Andrade et al., 2014a). Estudos realizados por Ellison, Hotamisligil, Lee, Rich-Edwards, Pang e Hall (2005), 
Olivennes, Golombok, Ramogida e Rust (2005) e por Sheard, Cox, Oates, Ndukwe e Glazebrook (2007), citados por Andrade e colaboradores (2014a), concluem que mães de gêmeos falam, mais frequentemente, de experiências difíceis, questionando-se mais sobre a parentalidade e colocam mais dúvidas. Sentem-se cansadas, revelando que as suas expetativas relativamente à maternidade eram diferentes, apresentando-se esta como um trabalho mais duro, menos gratificante e com mais dificuldades do que imaginavam. Em consequência, tendem a experienciar sintomas depressivos (Choi, Bishai, \& Minkovitz, 2009; Garel, Charlemaine, \& Blondel, 2006), e a perceber uma diminuição da sua qualidade de vida (Ellison et al., 2005, citados por Andrade et al., 2014a). Enquanto que com um bebé a mãe consegue estabelecer uma sincronia entre os períodos de vigília e de sono do filho, na situação gemelar, a repetição e o número de cuidados a ter são um entrave ao estabelecimento dessa ritmicidade conjunta. Nos primeiros dias, as mães de gémeos, submergidas pela sobrecarga dos cuidados a ter com as crianças e o esgotamento físico e psicológico, são privadas de uma parte dos prazeres e gratificações trazidos pelas reações dos recém-nascidos. Esta situação parece não ajudar a mãe a relacionar-se e a individualizar precocemente os seus bebés (Garel, Charlemaine, \& Blondel, 2006), observando-se, por vezes, confusões entre os filhos, particularmente no decorrer da amamentação. Em geral, como referem vários autores (eg., Anderson, \& Anderson, 1990; David, Azevedo, Russi, Berthoud, \& Oliveira, 2000), horários de amamentação, sono, banho e outras atividades rotineiras tendem a ser simultâneos ou sequenciados (termina de cuidar de um bebé e faz de seguida o mesmo com o outro, podendo alterar a ordem com que começa na vez seguinte), vestindo-os, muitas vezes, com roupas que os deixam mais parecidos. Embora as mães percebam que cada filho tem as suas necessidades, temperamento e padrões de interação, e que por isso devem responder-Ihes de forma individualizada, temem beneficiar um em prejuízo do outro e tendem a responder de forma similar aos dois. Ora, esta preocupação com a justiça entra em conflito com a tarefa de individualização (Anderson \& Anderson, 1990).

As mães/famílias que estão atentas a cada filho, e se mostram capazes de responder adequadamente às necessidades de cada um, são as que tendem a enfatizar as diferenças entre os gémeos e a adotar comportamentos diferenciadores: dão-lhes nomes diferentes, vestem-nos de forma diferente e enfatizam as suas diferenças comportamentais. Esta atitude facilita o desenvolvimento de uma relação individual com cada uma das crianças e 0 desenvolvimento de indivíduos distintos, capazes de realizações pessoais, num contínuo de transações com 0 seu meio. As que valorizam a igualdade e a justiça parental tendem a acentuar a gemelaridade dos filhos, nivelando as suas diferenças, seja pela escolha de roupas idênticas seja pela desvalorização das diferenças comportamentais. Como consequência, respondem de forma insuficiente e/ou inadequada às necessidades de um ou de ambos os bebés, 0 que pode afetar negativamente 0 desenvolvimento de relações individuais e a tarefa da individualização (Tourrette, Robin \& Josse, 1988).

A. Anderson e B. Anderson (1990), na sua teoria sobre a vinculação mãe-gémeos, referem que, quando os gémeos são dizigóticos, as mães tendem facilmente a polarizar as diferenças físicas dos seus filhos, numa fase inicial, e, mais tarde, as diferenças temperamentais. Só por volta dos quatro meses é que elas começam a ser capazes de se adaptarem às diferenças e às necessidades de cada gémeo, sendo que, apenas aos oito meses, 0 fazem de uma forma espontânea, já que, como refere uma mãe, eles passam a ser percebidos "mais como seres individuais do que como gémeos" (Anderson \& Anderson, 1990, p. 375).

Pelo exposto se percebe que é decorrente das competências percetivas e relacionais da mãe e do bebé, e da sua dança relacional, que o comportamento de vinculação se vai gradualmente desenvolvendo; e é da qualidade desta dança relacional que dependerá, a posteriori, o solo individual e a articulação de dois desenvolvimentos interligados, mas independentes. Não obstante, no caso das mães gemelares, estas têm como desafio acrescido estabelecer relações individuais com duas, ou mais, crianças, com a mesma idade, tendo em conta as suas necessidades idênticas, assim como as suas distintas personalidades, necessidades e padrões de interação, 0 que poderá comprometer a formação e o desenvolvimento do vínculo com o(s) filho(s) e a diferenciação dos mesmos. 


\section{OS PRIMEIROS MESES NA DANÇA RELACIONAL MÃE-BEBÉ(S): DESAFIOS DECORRENTES DA SITUAÇÃO GEMELAR}

\section{OBJETIVOS DE LA INVESTIGACIÓN}

Com o intuito de estudar a problemática enunciada, foi desenvolvida uma pesquisa que procurou investigar a interligação entre 0 tipo de relação vinculativa que a mãe estabelece com os filhos gémeos e alguns indicadores da diferenciação que a mesma faz em relação aos mesmos, desde o final da gravidez até aos seis meses pósparto.

Foram então definidas cinco questões de investigação:

1. Será que existe uma relação entre a capacidade da grávida gemelar fantasiar dois bebés distintos e a diferenciação pós-parto dos bebés reais?

2. Será que existe uma relação entre o tipo de ligação vinculativa mãe-bebés gémeos e a diferenciação posterior que a mãe faz dos mesmos?

3. Será que existe uma relação entre 0 sexo dos bebés e a diferenciação/individuação dos bebés reais, no pós-parto, aos três e aos seis meses?

4. Será que existe uma relação entre a zigotia e a forma como a mãe perceciona os seus filhos (como par com características e necessidades idênticas ou como seres individuais com características e necessidades diferentes)?

5. Será que existe uma relação entre a capacidade materna de perceber dois bebés com características e necessidades diferentes e a promoção, por parte desta, de comportamentos e atitudes diferenciadoras?

A presente comunicação, centrando-se no período pós-parto, procura evidenciar e discutir os resultados que decorrem da segunda questão de investigação.

Dada a amplitude da questão e havendo o risco de se perderem informações pertinentes, procurou-se identificar possíveis variáveis que afetem direta ou indiretamente a questão em estudo. Foram, assim, consideradas, como variáveis: a idade gestacional; o peso à nascença; a existência de complicações clínicas durante o parto; a vivência materna do parto (em relação a si e aos bebés); a estadia dos bebés na incubadora; a primeira imagem dos bebés reais; a capacidade da mãe integrar o bebé real no bebé imaginário; a perceção materna das vantagens/ desvantagens da situação gemelar; e a perceção materna acerca da sua competência para satisfazer plenamente ou responder adequadamente às necessidades dos filhos.

\section{MUESTRA Y/O PARTICIPANTES}

A amostra, constituída por quarenta mães gemelares, foi obtida em unidades hospitalares do Distrito do Porto. Todas as mães eram portuguesas, com idades compreendidas entre os 16 e os 37 anos, aquando o nascimento dos gémeos, situando-se a média nos 27,3 anos. 87\% pertenciam a um meio sócio-económico-cultural médio ou desfavorecido e 24 eram primíparas.

\section{METODOLOGÍA Y/O INSTRUMENTOS UTILIZADOS}

Em termos de procedimentos, foram realizadas três entrevistas semi diretivas, de tipo clínico, uma após 0 parto e as outras aos três e aos seis meses de vida, nas residências familiares. Nestas alturas, procurou-se, ainda e sempre que possível, realizar uma observação do vestuário, mobiliário e brinquedos dos bebés, bem como da interação das díades.

Para a análise dos dados, optou-se por uma metodologia quantitativa, utilizando uma análise correlacional das variáveis. Utilizou-se para o tratamento estatístico o teste de Qui-Quadrado ( ${ }^{2}$ ), com um índice de significância para a análise estatística de $p<.05$. Nos casos em que se observava mais de $20 \%$ das frequências esperadas inferiores a 5, o que tornava a análise estatística não legítima, optou-se por diminuir ou combinar as categorias adjacentes e/ou aplicar o Teste de Fisher. Sempre que necessário, realizou-se uma análise descritiva dos resultados, apelando para a análise das percentagens. 


\section{PSICOLOGÍA, INFANCIA Y EDUCACIÓN}

\section{RESULTADOS ALCANZADOS}

A análise estatística realizada concluiu sobre a não existência de uma relação clara entre 0 tipo de ligação vinculativa mãe-bebés gémeos e a diferenciação posterior que a mãe faz dos mesmos. Ou seja, a qualidade da relação mãe-bebés parece não apresentar uma relação significativa com a discriminação dos bebés nas suas semelhanças físicas e temperamentais, assim como nas parecenças físicas e nas suas necessidades, nem afectar as vivências projectivas maternas em relação ao vestuário dos filhos, à separação ou não destes aquando da entrada na escolaridade obrigatória e ao seu desenvolvimento aos dois-três anos e aos seis anos de idade.

No que concerne às variáveis estudadas, apresentam-se de seguida aquelas que evidenciaram uma relação significativa.

No pós-parto, a qualidade da relação vinculativa parece ser afetada pelo/a:

. Modo como a mãe vivenciou o parto relativamente a si (Teste ${ }^{2} .004$ MS);

- Modo como a mãe vivenciou o parto relativamente aos bebés (Teste de Fisher .01 S);

. Existencia de complicações clínicas durante o parto (Teste de Fisher .05);

. Primeira imagem materna dos bebés reais (Teste de Fisher .0005 MS);

. Estadia dos bebés na incubadora (Teste ${ }^{2} .0005$ MS);

. Capacidade de a mãe integrar o bebé real no bebé imaginário (Teste ${ }^{2} .02 \mathrm{~S}$ );

. Perceção materna de que é capaz/incapaz de satisfazer plenamente as necessidades de cada criança (Teste $2.04 \mathrm{~S})$.

No pós-parto, a capacidade da mãe integrar o bebé real no bebé imaginário parece ainda estar associada com a perceção materna das vantagens/desvantagens da situação gemelar (Análise Descritiva A).

Aos três meses, não se encontrou uma relação significativa entre as variáveis estudadas e a qualidade da relação vinculativa.

Já aos seis meses:

. A perceção materna das vantagens/desvantagens da situação gemelar parece estar associada com a qualidade da relação mãe-bebés (Análise Descritiva A);

- A perceção materna acerca das suas competências para lidar adequadamente com ambos os bebés parece estar associada com a perceção materna das vantagens/desvantagens da situação gemelar (Análise Descritiva A).

\section{DISCUSIÓN}

A existência de complicações clínicas nos bebés durante o parto e o modo como este é vivenciado pela mãe, relativamente a si e aos bebés, parece afetar a qualidade da relação mãe-filhos no período pós-parto. A vivência negativa do momento do parto e a vinda ao mundo de crianças com problemas médicos parecem tornar presentes todos os receios, angústias e dúvidas que a mãe tinha sentido durante a gravidez, não apenas de ter crianças com algum problema médico, mas mesmo a morte de um ou ambos os bebés.

A estadia dos bebés na incubadora parece agravar estes receios, afetando negativamente 0 estabelecimento do vínculo afetivo. Tal poder-se-á dever ao facto de a estadia na incubadora obrigar não só a uma separação mãefilho(s), como também obrigar a mãe a confrontar-se com uma circunstância que viola todas as suas idealizações: em vez de crianças fortes e saudáveis de quem pode cuidar, depara-se com bebés fracos, muitas vezes doentes, entregues aos cuidados de profissionais e que apenas vê de tempos a tempos, dentro de uma incubadora. Assim, ao deparar-se com bebés que em nada são parecidos com os seus bebés imaginários, que apresentam perturbações do desenvolvimento e que até podem morrer, é invadida por sentimentos de frustração e de culpabilidade por ter gerado crianças frágeis; estessentimentos poderão comprometer o equilíbrio emocional e o comportamento materno. Não obstante, a relação entre as variáveis identificadas só foi visível no período pós-parto e não aos 


\section{OS PRIMEIROS MESES NA DANÇA RELACIONAL MÃE-BEBÉ(S): DESAFIOS DECORRENTES DA SITUAÇÃO GEMELAR}

3 e 6 meses. Ora, tal poderá ser indicador da capacidade de reinstalação de uma relação saudável resultado de um potencial terapêutico da própria relação.

A primeira imagem que a mãe tem dos seus bebés reais e a sua capacidade de integrá-los nos bebés imaginários afetam de modo determinante a qualidade da relação mãe-bebés no período pós-parto (Franco, 2016). Uma imagem positiva torna-se, para a mãe, fonte de gratificação e reforça o instinto maternal. Quando a mãe se depara com filhos que em nada são parecidos com os bebés imaginários, que apresentam perturbações do desenvolvimento e que até podem morrer, poder-se-á verificar desajustamentos ou desinteresse da mãe pelos bebés e estes, por não encontrarem um espaço psíquico disponível para eles na interação, podem perder o interesse em responder e em solicitar a mãe. Não obstante, como verificado no estudo, esta situação pode alterar-se ao longo do tempo, o que parece reforçar de novo a ideia da dimensão temporal para a ativação do potencial relacional da própria díade e para a função terapêutica que os bebés podem desempenhar junto das suas mães.

Sem uma rede de suporte (pais, família alargada, outros), as mães dizem que dificilmente conseguiriam aceitar e lidar com a situação gemelar. Briggs (Anderson \& Anderson, 1987) defende que uma boa rede de suporte é o melhor prenúncio de uma vinculação segura mãe-bebés.

\section{CONCLUSIONES}

0 nascimento de gémeos é, como referem Neifert e Thorpe (citados por Akerman \& Thomassen, 1992), uma experiência parental única que se inicia durante a gravidez e alcança proporções críticas após o parto, quando as mães são confrontadas com o cuidar de dois (ou mais) bebés. Compreende-se, assim, a importância de um apoio efetivo e coordenado, já que as mães têm de enfrentar uma multiplicidade de desafios. Ajudá-las, nomeadamente, a ultrapassarem as suas dúvidas e incertezas quanto às suas capacidades de cuidar dos bebés, encorajando-as e orientando-as quanto ao modo como podem alimentar, cuidar, estimular e relacionar-se com os seus bebés, e com cada um em particular, é fundamental para que se criem condições para o estabelecimento do vínculo afetivo.

Neste processo é essencial que sejam (re)ativadas as redes de suporte da família, pois, como referem Gottelieb e Briggs (citados por Anderson \& Anderson, 1987) é quase impossível que esta equilibre as suas necessidades com as necessidades das crianças, sem ajuda externa. Aliás, a pesquisa revela que uma boa rede de suporte parece ser o melhor preditor de uma vinculação segura mãe-bebés, ou seja, as fontes de suporte parecem exercer um efeito positivo na interação mãe-bebé, particularmente nos casos em que os bebés são prematuros (Crnic et al., 1986; Feiring et al., 1989, citados por Figueiredo, 1992) ou têm um temperamento difícil (Hann, 1989; Pianta, 1989, citados por Figueiredo, 1992).

Sabendo-se que uma relação gratificante deve ser recíproca e ter sincronia, é importante observar como é que a mãe e as crianças estão a contribuir para este sistema comum. Assim, os profissionais devem estar atentos se a mãe/ família atende mais um filho do que 0 outro, se chama um deles pelo nome e 0 outro não, se se refere constantemente aos gémeos como "gémeos", se se dirige negativamente e inadequadamente a um e positivamente ao outro, se percebe um como o "mau" e o outro como o "bom" e se estes comportamentos são consistentes ou se ocorrem apenas em dias em que a mãe/família se sente irritada com um deles. Se se observarem consistentemente respostas inadequadas com um ou ambos os gémeos, é importante sensibilizar a mãe/família para esta situação e elaborar programas de intervenção para alterar este comportamento. Não obstante, é importante transmitir a importância da dimensão temporal para a ativação do potencial relacional da própria díade e para a função terapêutica que os bebés podem desempenhar junto das suas mães.

Partindo do pressuposto de que as mães precisam de diferenciar os gémeos para se relacionarem com cada um individualmente, é importante que os profissionais, durante o período pós-parto, avaliem as capacidades maternas em reconhecer as competências particulares de cada criança, particularmente quando são gémeos monozigóticos. Se o profissional perceber dificuldades no desenvolvimento de uma relação individual com cada um dos gémeos, é importante intervir, ajudando a mãe a descobrir as diferenças físicas e temperamentais dos seus filhos, bem como as suas necessidades particulares (Anderson \& Anderson, 1987). Este processo é facilitado quando os gémeos são de sexo diferente ou apresentam diferenças físicas visíveis, funcionando estes como 


\section{PSICOLOGÍA, INFANCIA Y EDUCACIÓN}

elementos diferenciadores importantes que permitem à mãe estabelecer comparações precoces entre as crianças e individualizá-las.

A par do acompanhamento e suporte profissional, é importante que estas mães/famílias sejam incentivadas a partilhar as suas dúvidas e questões com outras mães/famílias gemelares ou a participarem em encontros que Ihes permitam, por um lado, discutir os mitos relacionados com os gémeos e as especificidades desta realidade particular, e, por outro, partilhar as suas expectativas, receios e vivências. Estes espaços de encontro podem promover, por si só, a emergência de interações mais adequadas, facilitando o processo de vinculação, bem como a tarefa materna/familiar da separação-individualização.

Pelo exposto se percebe que a família gemelar, e a mãe em especial, vivencia uma multiplicidade de desafios, dos quais se destaca a sua capacidade de olhar para as crianças como sujeitos individuais e de os tratar de forma diferenciada, favorecendo, deste modo, a identidade e a individualidade de cada um e estabelecendo um vínculo satisfatório e sadio com ambos.

\section{REFERENCIAS BIBLIOGRÁFICAS}

Anderson, B., \& Anderson, A. (1987). Assessing families with problems attaching to twin infants. In M. Leahey \& L. Wright (Eds.), Families and psychosocial problems (pp. 64-77). Pennsylvania: Aprenghouse Publishers.

Anderson, B., \& Anderson, A. (1990). Toward a substantive theory of mother-twin attachment. Maternal Child Nursing, 15(6), 373-377.

Andrade, L., Martins, M., Angelo, M., \& Martinho, J. (2014a). A família na vivência da gemelaridade - Revisão sistemática. Texto \& Contexto Enfermagem, 23(3), 758-66.

Andrade, L., Martins, M., Angelo, M., \& Martinho, J. (2014b). A saúde mental na parentalidade de filhos gémeos - Revisão da literatura. Revista Portuguesa de Enfermagem de Saúde Mental, Especial 1, 109-116.

Bakeman, R., \& Brown, J., (1977). Early interaction: consequences for social and mental development at three years. Confrontations Psychiatriques, 16, 83-124.

Blondel, B. (2012). Augmentation des naissances gémellaires et conséquences sur la santé. Journal de Gynécologie Obstétrique et Biologie de la Reproduction, 38(8S1), 7-17.

Bryan, E. (2002). Educating families, before, during and after multiple birth. Seminars in Neonatologie, 7(3), 2416.

Charlemain, E., \& Garel, M. (2009). Difficultés psychologiques des mères d'enfants multiples: de la grosssesse aux premières années. In M. Dehan \& D. Lacombe (Eds.), Les jumeaux et leur pédiatre (s/p). Paris: Doin Editeurs.

Choi, Y., Bishai, D., \& Minkovitz, C. (2009). Multiple birth are a risk factor for postpartum maternal depressive symptoms. Pediatrics, 123(4), 1147-1154.

Colpin, H., Munter, A., \& Nys, V. (2000). Pre and postnatal determinants of parenting stress in mothers of one year-old twins. Marriage Family Review, 30(1), 99-107.

David, D., Azevedo, E., Russi, E., Berthoud, C., \& Oliveira, A. (2000). Tríade de contato íntimo: Apego entre mãe e filhos gêmeos. Revista Biociência, 6(1), 7-63.

Dias Cordeiro (1994). A saúde mental e a vida (3르 ed.). Lisboa: Salamandra (obra original publicada em 1982).

Figueiredo, B. (1992). Contextos de educação e desenvolvimento: A interacção mãe-bébé (Dissertação das Provas de Aptidão Científica e Capacidade Pedagógica não publicada). Universidade do Porto, Porto, Portugal.

Franco, V. (Org.) (2016). Contributos psicodinâmicos para a intervenção precoce na infância. Évora: Edições Aloendro.

Garel, M., Charlemaine, E., \& Blondel, B. (2006). Conséquences psychologiques des naissances multiples. Gynécologie Obstétrique \& Fertilité, 34(11), 1058-63.

Glazebrook, C., Sheard, C., Cox, S., Oates, M., \& Ndukwe, G. (2004). Parenting stress in first-time mothers of twins and triplets conceived after in vitro fertilization. Fertility and Sterility, 81(3), 505-511. 


\section{OS PRIMEIROS MESES NA DANÇA RELACIONAL MÃE-BEBÉ(S): DESAFIOS DECORRENTES DA SITUAÇÃO GEMELAR}

Lester, B., Hoffman, J., \& Brazelton, T. (1985). The rythmic structure of mother-infant interaction in term and preterm infants. Child Psychology, 56, 15-27.

Manso, P., Vaz, A., Taborda, A., \& Silva, I. (2011). Coronocidade e complicações perinatais na gravidez gemelar. Casuística de 10 anos. Acta Médica Portuguesa, 24, 695-698.

Robin, M., \& Josse, D. (1987). Quelques aspects de la relation mère-enfant à la suite d'une naissance gémellaire. Neuropsychiatrie de l'Enfance et de l'Adolescence, 35(8-9), 369-377.

Rodrigues, D., Fernandes, A., Silva, R., \& Rodrigues, M. (2006). 0 domicílio como espaço educativo para o autocuidado de puérperas: binômio mãe-filho. Texto \& Contexto Enferm, 15(2), 77-86.

Rodrigues, C., Branco, M., Ferreira, I., Nordeste, A., Fonseca, M., Taborda, A., Silva, I., \& Almeida, M. (2005). Epidemiologia da Gestação Múltipla - Casuística de 15 anos. Acta Médica Portuguesa, 18, 107-111.

Siddiqui, F., \& McEwan, A. (2007). Twins. Obstetric, Gynaecology and Reproductive Medicine, 17(10), 289-296.

Silva, J., Cecatti, J., Pires, H., Passini Jr, E., Parpinelli, E., \& Pereira, B. (2003). Assistência à gestão e parto gemelar. Revista Ciências Médicas, 12(2), 173-183.

Souza, L., Madi, J., Araújo, B., Zatti, H., Madi, S., Lorencetti, J., \& Marcon, N. (2010). Características e resultados perinatais das gestações gemelares (1998-2007). Revista AMRIGS, 53(2), 150-155.

Tourrette, C., Robin, M., \& Josse, D. (1988). Les pratiques éducatives des mères de jumeaux: Une investigation par l'analyse factorielle des correspondences. Année Psychologique, 88(4), 545-561.

Van der Zalm, J. (1995). Accommodating a twin pregnancy: maternal processes. Acta Geneticae Medicae et Gemellelogiae, 44, 117-133.

Veiga, S. (1997). Vinculação e separação-individuação em díades mãe-bebés gémeos (Tese de Mestrado não publicada). Universidade de Coimbra, Coimbra, Portugal. 